\title{
Contexto actual de los sistemas de costes de la calidad desarrollados y aplicados a proyectos de construcción: la necesidad de medición de la calidad en el diseño
}

\author{
Present context of quality costs system developed and \\ applied to construction projects: the necessity of \\ measurement of quality in design
}

\author{
L. A. Dzul ${ }^{(*)}$, S. Gracia ${ }^{(*)}$
}

RESUMEN

Este trabajo presenta un análisis de las metodologías desarrolladas para el seguimiento y control de los costes de la calidad en proyectos de construcción, proporcionando un contexto actual de esta herramienta de calidad en esta industria. Se realizó una revisión bibliográfica y análisis de las publicaciones sobre sistemas de costes de la calidad aplicados a proyectos de construcción (QPMS, QPTS, QCM, CQCQS, PCM y PROMQACS). Estos enfoques propuestos se han venido desarrollando desde los años ochenta. Dichos sistemas muestran un aspecto común referente a su aplicación en la fase del proyecto; se realiza a partir del inicio de la construcción en el sitio, y no toman en cuenta los costes de la calidad dentro de los procesos de diseño. Para complementar este contexto, se realizó una revisión de publicaciones de prácticas reales de los procesos de diseño, donde se destacan los principales problemas durante dicha fase. Este trabajo muestra un entendimiento de la necesidad de la medición de los costes de la calidad en el diseño de proyectos $y$, por consiguiente, las limitaciones de los sistemas propuestos actualmente de costes de calidad en la industria de la construcción. Destacando la importancia de una herramienta de planificación de la calidad en una línea de mejora continua, en un entorno donde el número de publicaciones sobre costes de la calidad es limitado, debido al número y complejidad de los procesos típicos de un proyecto de construcción.

197-2

Palabras clave: calidad, costes de la calidad, proyectos de construcción, diseño.

\section{SUMMARY}

This paper presents an analysis of the approaches developed for measuring and tracking the quality costs in construction projects, providing a current context of this quality management tool. A literature review of currents publications on generic models of quality costs applied on construction projects (QPMS, QPTS, QCM, CQCQS, PCM y $P R O M Q A C S)$ was performed. These approaches have been developed since the eighties. It was observed that these approaches share a common aspect regarding the timing of their application during the project; which begins at the start of construction on site, without taking into account the failures and quality management costs within the design process. In order to enhance this context, a literature review was made to evaluate actual practices during the design process. This work demonstrates the need to measure quality costs during project design as well as the limitations of the proposed quality costs systems currently applied in the construction industry. Emphasis is made about the importance of implementing a quality management tool in line with continual improvement approaches, in a context where the number of publications on quality costs is limited, given the number and complexity of the typical processes of a construction project.

Keywords: quality, quality costs, construction projects, design.

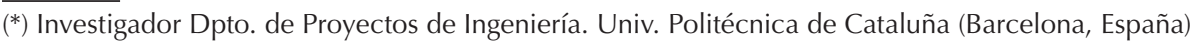

(**) Profesor Titular. Univ. Politécnica de Cataluña (Barcelona, España)

Persona de contacto/Corresponding author: luis.alonso.dzul@upc.edu (L. A. Dzul) 


\section{INTRODUCCIÓN}

La calidad en la construcción debe ser un deseo buscado por todas las organizaciones involucradas con la actividad; por lo tanto, ésta debería formar parte de la misión empresarial. Su logro requiere identificar el camino apropiado, siendo necesario establecer las estrategias y tácticas más adecuadas para cada organización. Las exigencias que se reclaman desde la industria de la construcción en la actualidad, obligan el desarrollo de nuevas herramientas de gestión, que permitan potenciar los recursos, principalmente en países en donde la inversión es limitada. Hay un factor que hace la diferencia entre la manera costosa y la forma provechosa de alcanzar calidad, ese factor son los costes de la calidad (1). La gestión de los costes de la calidad permite a las organizaciones precisar las fuerzas y las debilidades de un sistema de gestión de la calidad. En el caso de la industria de la construcción, se estima que los costes de la calidad se encuentran entre un 8 a 15\% de los costes de la construcción total (2).

Los costes de la calidad son aquellos gastos que se refieren a la corrección de fallos, a la verificación de procesos, a medidas que se tienen que tomar para obtener un mejor producto; por lo que al proporcionar una visión de los alcances benéficos del adecuado control de los costes de la calidad, se daría una herramienta para que dichas empresas, incluso sin certificar el aspecto de la calidad, tengan mayor y creciente calidad en sus procesos $y$, por consiguiente, mayor productividad como empresa (3).

En la actualidad la teoría sobre los modelos genéricos de costes de la calidad está documentada, pudiéndose encontrar información teórica y práctica; de igual manera, existe un interés académico sobre este tema; sin embargo, a pesar de la importancia cuantitativa de dichos costes en los proyectos de construcción, existe un tratamiento limitado con respecto a otras industrias.

El propósito de este trabajo es plantear el desarrollo de los sistemas de costes de la calidad en los proyectos de construcción, desde las primeras propuestas hasta la actualidad, destacando sus características principales, relaciones, alcance y limitaciones. La metodología se basó en una revisión de la literatura y una discusión de las evidencias del éxito de la aplicación de modelos genéricos de costes de la calidad en general y de los modelos planteados específicamente para proyectos de construcción, destacando sus limitaciones en la fase del diseño de los proyectos. De esta manera, el contexto se complementó haciendo un breve planteamiento de las prácticas actuales en el diseño de proyectos de construcción. Así, se plantea y promueve una línea de investigación, en busca del desarrollo y ordenación de las variables actuales que definen el desarrollo actual de herramientas de gestión para la industria de la construcción.

\section{EVIDENCIA BIBLIOGRÁFICA DEL ÉXITO DE MODELOS GENÉRICOS DE COSTES DE LA CALIDAD}

Dentro de las revisiones de los modelos genéricos de costes de la calidad, en un contexto de resumen de actualidad en su momento, están Hwang y Aspinwall (4), que analizaron los diversos modelos de costes de la calidad actuales, comparando las ventajas y las debilidades de cada modelo, destacando su uso en diversas áreas.

Schiffauerova y Thomson (5) propusieron un resumen de los autores que estudiaron los modelos de costes de la calidad, agrupando los diversos modelos en 5 categorías, de acuerdo a las categorías de costes o actividades manejados. Gracia y Dzul (3) presentaron cronológicamente los estudios realizados en este tema, basado en el trabajo de Schiffauerova y Thomson (5), agregando un modelo propuesto por Weheba y Elshennawy (6), encontrado en su revisión; permitiendo visualizar de manera más práctica el modelo de costes de la calidad más estudiado y desarrollado.

A pesar de los datos publicados, los costes de la calidad no es un concepto ampliamente usado, ya que las grandes compañías a pesar de que afirman evaluar los costes de la calidad, sólo un pequeño número de ellas mide realmente los resultados de programas de mejora de la calidad (5). La mayoría de los ejemplos publicados en este tema, confirman que los procesos de medición de costes, producen una gran reducción de los costes de la calidad de la empresa.

Sin embargo, se puede observar que los casos documentadas corresponde a un cierto sector industrial, tal como: telecomunicaciones, tecnología de la informática, electrónica, software, servicios financieros, industria del acero, equipamiento, e incluso el sector aeroespacial. Resaltando la carencia de datos de industrias importantes en la economía, tal es el caso de la industria de la construcción.

La mayoría de las compañías han empleado la universalmente aceptada estructura de costes de la calidad: costes de Prevención, Evaluación y Fallos (PEF), planteada recientemente en el contexto de las empresas constructoras por Gracia y Dzul (3). Hay algunos casos 
evidenciados que destacan, tal es el caso de la compañía multinacional ITT EuropeBelgium, donde los ahorros por el programa de mejora de costes de la calidad, totalizaron más de 150 millones de dólares en cinco años; otros ejemplos se encuentran en la industria de telecomunicaciones, siendo el caso de la empresa norteamericana United Technologies (Essex Group) con un aumento del $26 \%$ en su productividad; o la empresa de software Raytheon's Electronic Systems, con un aumento del mismo parámetro de un $170 \%(5)$.

Recientemente se planteó un trabajo sobre la importancia de los costes de calidad en las decisiones de la organización en las empresas certificadas con la norma ISO 9000 (7); los objetivos del trabajo eran analizar los modelos de costes de calidad y los sistemas, metodologías y herramientas de gestión de la calidad que utilizan las empresas certificadas en la norma ISO 9000, de la Comunidad Valenciana en España. En esta investigación se observó que el 43\% de las empresas certificadas, una vez deducidos los gastos por la implantación del sistema de calidad, incluidos los costes de certificación, se habían reducido los costes totales de la empresa al implantar un sistema de calidad. El sector de la Construcción fue el segundo en reducir sus costes, el cual representaba el 16,9\% del total de empresas certificadas.

Otro dato a destacar de esa investigación (7) era cómo las empresas u organizaciones median los costes de calidad, de acuerdo con las diferentes clasificaciones mayoritariamente aceptadas. La categoría de costes de la calidad que era más medida por las empresas, era la de los fallos internos, seguida por las categorías de fallos externos, costes de prevención y de evaluación. Se llegó a la conclusión de que la implantación de los sistemas de calidad, llevaba a las empresas a implantar sistemas de control de costes de calidad.

El Instituto Tecnológico de Galicia (ITG), en colaboración con otras instituciones promueve actualmente el proyecto Costes de Calidad en la Construcción (CCCON) en Galicia, España; el objetivo del proyecto es incrementar la competitividad de las empresas del sector de la construcción mediante el diagnóstico de la eficacia y la eficiencia de la gestión empresarial basado en las teorías del análisis de los costes de calidad.

El proyecto está dirigido a 10 pymes del sector de la construcción y afines de la Comunidad Autónoma de Galicia. Se hace referencia a este estudio, ya que se observa la importancia y relevancia de la aplicación de esta herramienta; de igual manera, la validez del desarrollo de nuevas teorías específicas para el sector, que se adapten a las necesidades propias y actuales (8).

A pesar de que el modelo más implementado en la práctica es el enfoque clásico PEF y se emplea como concepto básico; otras categorizaciones de costes de la calidad han sido usadas con éxito. Los sistemas de costes de la calidad deberían ser diferentes de compañía a compañía y deberían ajustarse a las necesidades de la empresa. Schiffauerova y Thomson (5) sugieren una investigación adicional respecto a cómo debería ser conducida la reducción de los costes de la calidad; de igual manera plantear la manera en que las empresas deberían tomar decisiones con respecto a la mejora de la calidad, es decir, estudios más detallados en recolección y medición de costes de la calidad.

\section{SISTEMAS DE COSTES DE LA CALIDAD EN PROYECTOS DE CONSTRUCCIÓN. REVISIÓN DE MODELOS}

Recientemente se realizó un estudio sobre la implementación de sistemas de gestión de la calidad en las industrias de la construcción de los Estados Unidos de América y de Hong-Kong, haciendo énfasis en la norma ISO 9000:2000; a través de una encuesta a empresas de ambos países y profundas entrevistas. Entre las principales conclusiones obtenidas, estuvo la de que el éxito de cualquier sistema de gestión de la calidad, depende principalmente de la alta gerencia y en cómo valoran a los clientes (1); de igual manera, se enfatizó en que para determinar la eficacia de los sistemas de gestión de la calidad, es esencial la cuantificación de la mejora de calidad.

Las empresas de mencionados los países, empleaban diversas herramientas para medir la mejora de la calidad, pero de acuerdo con la información obtenida en la investigación, se observó que estas medidas de calidad eran principalmente para supervisar y solamente con el propósito de registro. Lo anterior, resalta la necesidad de herramientas de gestión de calidad, que proporcionen información directa sobre la mejora de procesos y la satisfacción del cliente; tal es el caso de la aplicación de un modelo de gestión de costes de la calidad.

Los costes de la calidad en la industria de construcción en su totalidad son relativamente altos en términos de los costes totales del proyecto (9). Una revisión extensa de la literatura, demuestra que es reducido el número de publicaciones sobre la forma en que los costes de la calidad podrían ser determinados en la industria de la construcción (10). En esta investigación, se encontraron y analizaron los sistemas de gestión de costes 
de la calidad para proyectos de construcción, desarrollados hasta ahora. La tabla 1 muestra un resumen de ellos.

En 1987 el Quality Management Task Force del Construction Industry Institute (CII) desarrolló el Quality Performance Management System (QPMS) como una herramienta para el análisis cuantitativo de ciertos aspectos relacionados a la calidad $(9-11,14)$. El coste de la calidad es definido como el costo de corrección de desviaciones (reprocesos o fallos) más el costo de las actividades de gestión de la calidad. Este sistema supone que los costes de la calidad pueden ser adecuadamente rastreados usando 11 causas de reprocesos y 15 actividades de gestión de calidad. Se probó en 9 proyectos industriales, produciendo un coste promedio de reprocesos o fallos del $12,4 \%$ del coste del proyecto (10). Este sistema era simple y flexible, pero no consideraba el efecto de los costes de fallos en el tiempo. Además, el sistema no identificó las causas específicas de fallos (9).

El Quality Performance Tracking System (QPTS) de K. Davis es una versión renovada del modelos QPMS (9-11) fue desarrollado para caracterizar los costes de la calidad de analizarlos cuantitativamente y rastrear las desviaciones. En este modelo, los costes de desviación incluían reprocesos, el impacto, la responsabilidad y el trabajo de garantía. Davis observó que la definición de gestión de calidad variaba de una empresa a otra, y la distinción entre práctica de diseño y gestión de calidad era confusa (17). La ruta específica de desviaciones no fue registrada por el QPTS.
Abdul-Rahman (17) desarrollo la Quality Cost Matriz (QCM) para recopilar los costes de la calidad en la etapa de construcción; al poner de manifiesto las limitaciones del QPTS (9, 19). Cabe destacar la aplicación de este sistema sólo en la fase de ejecución del proyecto. Esta matriz consideraba el efecto de las fallas en el tiempo, el coste de la aceleración del trabajo y la ruta especifica de las no conformidades; sin embargo, no considero el origen de las desviaciones. El modelo se basa recopilar los costes de fallos o no conformidad, sin capturar los costes de evaluación o prevención; así como el origen de las desviaciones. Se probó en dos proyectos de construcción, y el total de los costes de no conformidad fueron el 5\% del coste total (5). Low e Yeo (2) de la Universidad Nacional de Singapur desarrollaron el Construction Quality Cost Quantifying System (CQCQS); este sistema es una matriz de documentación que adopta el modelo PEF de costes de la calidad.

La característica principal de este modelo es el uso de una codificación para clasificar los diversos elementos bajo un encabezado Ilamado "trabajos comprometidos", que podían ser obtenidos bajo una estructura de desglose de trabajo (WBS). Al igual que la QCM de Abdul-Rahman no se cuantificarón los costes de prevención y evaluación, ni el origen de las desviaciones.

Aoieong et al. (10) propusieron una medición de los costes de la calidad bajo un enfoque de procesos; un enfoque alternativo basado en el modelo de costes de procesos (PCM) de la norma BS 6143, considerando costes de conformidad y no conformidad; $y$, de esta

Tabla 1

Sistemas de gestión de costes de la calidad en proyectos de construcción

\begin{tabular}{|c|c|c|}
\hline Autor & $\begin{array}{c}\text { Sistemas de gestión de costes de la calidad en } \\
\text { proyectos de construcción }\end{array}$ & $\begin{array}{l}\text { Referencias } \\
\text { bibliográficas }\end{array}$ \\
\hline $\begin{array}{c}\text { CII Quality } \\
\text { Management } \\
\text { Task Force-1987 }\end{array}$ & QPMS (Quality Performance Management System) & $\begin{array}{l}9,10,11,12 \\
13.14\end{array}$ \\
\hline K. Davis-1987 & QPTS (Quality Performance Tracking System) & $\begin{array}{c}9,10,11,15 \\
16\end{array}$ \\
\hline $\begin{array}{l}\text { Abdul-Rahman- } \\
1993\end{array}$ & QCM (Quality Cost Matrix) & $\begin{array}{c}9,10,11,17 \\
18,19\end{array}$ \\
\hline Low y Yeo-1998 & $\begin{array}{c}\text { CQCQS (Construction Quality Cost Quantifying } \\
\text { System) }\end{array}$ & $2,9,10$ \\
\hline $\begin{array}{l}\text { Aoieong et al.- } \\
2002\end{array}$ & QCPCM ( Quality Costs- Process Costs Model) & 10,20 \\
\hline $\begin{array}{l}\text { Love e Irani- } \\
\quad 2003\end{array}$ & $\begin{array}{l}\text { PROMQACS (Project Management Quality Cost } \\
\text { System) }\end{array}$ & $9,19,21,22$ \\
\hline Otros enfoques & $\begin{array}{l}\text { Metodología basada en la adecuación del modelo } \\
\text { PEF, de acuerdo a las características de la industria. } \\
\text { Puntualizando en el estudio de los costes de fallos. } \\
\text { Propuesta de metodología basada en el modelo } \\
\text { PEF y el enfoque de Barber et. al., } 2000 \text {. } \\
\text { Puntualizando en el estudio de los costes de } \\
\text { prevención y evaluación. }\end{array}$ & 19,23 \\
\hline
\end{tabular}


manera, facilitar uno de los objetivos de la gestión de la calidad, la mejora continua. Realizaron una revisión de los sistemas de Davis, Abdul-Rahman, Low y Yeo y otros, mencionados anteriormente; destacando que dichos sistemas reconocían los componentes de los costes de la calidad, pero no direccionaban las causas o rutas de desviaciones indeseadas.

El modelo de costes de procesos de costes de la calidad (QCPCM), se basa en medir los costes de conformidad y no conformidad de las actividades claves en los procesos que requieren mejora continua, bajo un enfoque de gestión de calidad total (TQM). Realizaron entrevistas a especialistas y empresarios reconocidos de la industria de la construcción, llegando a la conclusión de que un sistema de costes de la calidad PEF, por si solo, resultaba complicado de implantar debido a la complejidad de la industria de la construcción. En este enfoque los costes de la calidad son llamados costes de procesos, los cuales se dividen en: costes de conformidad (COC) y costes de no conformidad (CONC). Los COC son los costes intrínsecos requeridos para proveer el producto final tal como es especificado y requerido, los CONC son los costes del tiempo de retraso, materiales, recursos y otros costes asociados a la rectificación de la insatisfacción del producto.

El modelo QCPCM proponía medir los costes de la calidad de procesos específicos que requerían mayor atención, y no concentrándose en la medición del coste total de la calidad del proyecto, que resulta ser muy complejo. Destacaron su aplicabilidad en el contexto de norma ISO 9000, como herramienta de medición de la mejora continua y resaltaron que debido a limitaciones de tiempo el modelo no fue probado, recomendando esta variable como futura línea de desarrollo. A diferencia de una producción lineal en la industria de manufactura, los procesos de construcción son más complicados debido al gran número de partes involucradas y la singularidad de cada actividad en un proyecto.

Love e Irani (9) propusieron el Project Management Quality Cost System (PROMQACS) para la determinación de los costes de fallos o reprocesos en proyectos de construcción. Este enfoque había sido propuesto y desarrollado por el autor en diversas publicaciones, así como temas afines al mismo $(21,22)$. El sistema desarrollado fue probado en dos casos de estudio en proyectos de construcción para determinar los costes y causas de reprocesos o fallos ocurridos.

El sistema se basó en la información obtenida en una investigación realizada a una empresa constructora líder en la implementación en sistemas de calidad, con la colaboración de los consultores y organizaciones involucradas con los proyectos. La información obtenida sobre los costos de reprocesos o fallos se clasificó en módulos que correspondían a los siguientes aspectos: el problema especifico, ajustes hechos por el contratista, la causa específica del fallo, el tiempo no productivo, clasificación de los costes de reprocesos, el coste directo del fallo. En relación a la clasificación de los costes de fallos, proponía una categoría alternativa de tres niveles (9), adaptada de la propuesta J. L. Burati. El primer nivel de clasificación se refería a la fase del proyecto en la que se originó el fallo: diseño o construcción; el segundo nivel, al tipo de reproceso: cambios, errores, omisiones o daños (en la fase de construcción); el tercer nivel se refería solamente al origen de los cambios realizados tanto en la fase de diseño o construcción.

La información fue recogida desde la fecha de inicio de la construcción hasta el final del periodo de responsabilidades por posibles defectos, principalmente durante el proceso de producción en el sitio de obra. Love e Irani sugirieron que el PROMQACS podría ser usado específicamente para monitorear el progreso de los cambios del cliente y proveer un mecanismo a los directores de proyectos para el control de cambios antes de que los fallos o reprocesos sean más caros. Love e Irani destacan la dificultad que representa la accesibilidad a los datos, necesitando que todas las partes involucradas participen en facilitar dichos datos; así como la necesidad de actividades de gestión y planificación de la calidad por parte de la administración de la empresa.

EI PROMQACS consideraba los fallos a partir de la construcción y los referentes a diseño se relacionaban a: cambios hechos por el cliente, peticiones del contratista, del futuro ocupante, de algún proveedor o de revisiones de rediseño por mejora iniciados por el contratista o subcontratista. Todo esto ocasionaría reprocesos por el rediseño. Sin embargo, hay que considerar que un control de costes de calidad durante la fase propia de diseño, reduciría estos reprocesos. Tang et al. (20) retomaron el modelo QCPCM, publicando el resultado de su investigación. EL estudio describía dos casos en los que se empleo dicho modelo, para la medición de los costes de la calidad en dos proyectos de construcción. Los estudios revelaron que el QCPCM era factible, práctico y fácil de utilizar. Resaltaron también que el modelo era un medio de alcanzar la mejora continua, ya que su enfoque de procesos permitía calcular los costes de la calidad de un proceso de construcción en particular; así como su conveniencia para la industria de construc- 
ción. Destacaron las desventajas del modelo tradicional PEF para el uso en la industria de construcción.

Este método no fue usado para calcular los costes de la calidad de un proyecto completo, sino para un proceso en particular, considerando el lineamiento de la Norma ISO 9000, en relación al enfoque de procesos y mejora continua, dentro del concepto del Total Quality Management (TQM). El proceso elegido para probar dicho método, fue el de elaboración de elementos de hormigón en dos proyectos (una edificación de 38 niveles y la construcción de pilas, un proyecto de ingeniería civil), ya que consideraron que era la actividad más común en cualquier proyecto de construcción. En este estudio se plantearon dos principios básicos para la aplicación del QCPCM. Primero, la aplicación del modelo debe ser fácil y no complicada para el entendimiento y uso del personal en sitio, con preparación ordinaria o nivel técnico; segundo, el modelo debería funcionar como una herramienta para la mejora continua de procesos.

En el caso del edificio 38 plantas, los costes de no conformidad, disminuyeron en el transcurso del estudio, de un $0,48 \%$ a un $0,43 \%$ de los costes totales del proceso. En el proyecto de ingeniería civil, el modelo QCPCM demostró que los CONC bajaron drásticamente de un 3,55\% de los costes totales del proceso a un insignificante $0,03 \%$. La mejora continua mostrada en este estudio, fue la mejora de un proceso en específico dentro de un proyecto. A pesar de que los ejemplos en esa publicación se relacionaban con el proceso de hormigón solamente, el modelo QCPCM podría haberse aplicado a cualquier proceso de la construcción de una manera similar. Tang et al. sugirieron el uso del QCPCM para los procesos de gestión.

Con respecto a este último punto, se puede comentar que si la aplicación de este modelo en el proceso de diseño hubiera generado las mismas cifras de costes de no conformidad; de nuevo se vuelve a resaltar la conveniencia y necesidad de la aplicación integrada de medición de los costes de calidad en el ciclo de vida del proyecto.

De igual manera se han propuesto otros enfoques basados en el modelo PEF, desarrollados en consideración a las características propias de los proyectos de construcción; tal es el caso de Barber et al. (23) y Hall y Tomkins (19). Barber et al. desarrollaron una metodología para medir el coste de fallos de la calidad en dos proyectos importantes de caminos; basado en gran parte sobre un método de "observación de trabajo" para la identificación y registro de datos. Dicho enfoque de investigación no tenia ningún precedente.

El personal en el sitio de la obra (ingenieros, encargados de obra, personal clave) observaba detalladamente por un periodo de tiempo, registrando los problemas de calidad encontrados. En la investigación publicada (23) se muestra cómo los datos iníciales fueron recogidos y colocados según su categoría en grupos definidos, así como la manera en que dichos costes eran estimados para cada una de dichas categorías. Llegando a la conclusión de que si los proyectos examinados fueran típicos, el coste de fallos podría ser un porcentaje significativo de los costes totales, y que los medios convencionales de identificarlos, no pueden ser de mucha confianza; refiriéndose a la clasificación de fallos internos y fallos externos del modelo PEF. De esta manera, sólo los costes directos de reprocesos para los fallos, fueron incluidos en este estudio; del coste del presupuesto semanal de las áreas especificas de trabajos estudiados, los costes de fallos representaron el $16 \%$ y el $23 \%$ del presupuesto semanal, en cada uno de los dos proyectos.

Hall y Tomkins propusieron una metodología para evaluar, de una manera completa, los costes de la calidad para proyectos de construcción, mostrando los resultados de un proyecto de edificación en el Reino Unido, en el cual la metodología fue probada. Dicha metodología desarrollada, se basó en el modelo de costes de la calidad PEF, definida por la norma Británica BSI 6143 parte 2, pero modificada en una línea con la metodología descrita por Barber et al.

Hall y Tomkins resaltaron la demanda de estudios en el tratamiento de los costes de la calidad en proyectos de construcción; destacando que los estudios propuestos hasta el momento no tomaban en cuenta la contribución de los costes de calidad de prevención y evaluación, limitando su análisis en el impacto de los costes de la calidad totales. De acuerdo a Tang et al. (20) éste era el único trabajo basado en el modelo PEF, que podría ser considerado como comprensivo y de éxito para la evaluación de los costes de la calidad; atribuyéndolo a que consideraron que el proyecto estudiado era muy pequeño (aproximadamente 2 millones de euros); sin embargo, Tang et al. resaltaron que la mayoría de los proyectos de construcción son más grandes y complejos, destacando que no es recomendable el empleo del modelo PEF. EI ejercicio de aplicación de la metodología de Hall y Tomkins, fue limitado al proceso de construcción, excluyendo los costes de la fase de diseño. Los resultados mostraron que el coste total de fallos fue del 5,84\% y el de los costes de prevención, evaluación y 
otros relacionados, del $12,68 \%$, en relación al coste total del proyecto.

\section{DISCUSIÓN DE LOS MODELOS PLANTEADOS}

Existen diversas publicaciones de aplicaciones de dichos modelos de sistemas de gestión de costes de la calidad en proyectos de construcción $(3,11,14,18,19-23)$; en los cuales se resalta la importancia de su aplicación y beneficios para la industria de la construcción, cuya naturaleza de procesos no lineal, hacen, de la medición de costes de la calidad, un tema a desarrollar. La figura 1 muestra la cronología de los modelos QPTS, QPMS, QCM, CQCQS, QC-PCM y PROMQACS; donde se observa el reciente estudio de la aplicación de esta herramienta de calidad en la gestión de proyectos de construcción.

Los sistemas de gestión de costes de la calidad para proyectos de construcción revisados con anterioridad, denotan un aspecto común, referente a la fase de aplicación de los mismos; es decir, la aplicación se realiza principalmente, a partir de la fase de ejecución del proyecto. Se hace referencia al diseño, principalmente como causa de fallo; es decir, como generadora de fallos. Es sabida la importancia de un correcto diseño, ya que evita errores en las fases posteriores; sin embargo, los sistemas de medición de los costes de la calidad no toman en cuenta los costes de gestión de la calidad y de fallos, de manera puntual, durante los procesos de diseño.
Las diferencias y similitudes entre los distintos sistemas de gestión de costes de la calidad en proyectos de construcción, se pueden observar en la tabla 2 (pág. siguiente), en relación a diversas características $(9,10,20)$.

Reforzando la idea planteada anteriormente, Abdul-Rahman (11) propuso la medición de los costes de la calidad en todos los niveles del proyecto. Hall y Tomkins (19) resaltaron la poca consideración que se tenía de los costes de la calidad en el diseño de proyectos de construcción, por parte de los estudios previos propuestos; Tang et al. (20) propusieron por primera vez, como trabajo de futuro desarrollo, la medición de los costes de la calidad en los procesos de diseño en proyectos de construcción.

\section{EL DISEÑO EN PROYECTOS DE CONSTRUCCIÓN}

Como se observó anteriormente, la aplicación de los modelos de sistemas de costes de la calidad están limitadas a la fase de ejecución del proyecto. El diseño es una parte integradora de la industria de la construcción, ya que la calidad de los diseños es la clave para el éxito del proyecto en general y para la fase de construcción en particular. Chuang y Tsai (24) y Wang et. al (25), realizaron un estudio completo acerca de la gestión profesional de la construcción en Taiwán, obteniendo como resultado la generalización de 6 problemas críticos en la planeación de proyectos y 15 en el diseño. Esta base de conocimiento, a
Desarrollo cronológico de los sistemas los costes de la calidad aplicados en proyectos de construcción.

\section{Publicaciones desarrollando o analizando los modelos}

Metodologías para la evaluación y seguimiento de los costes de la calidad en proyectos de construcción

Tang et al. (2004) 
Tabla 2

Diferencias y similitudes entre los distintos sistemas de gestión de costes de la calidad en proyectos de construcción

\begin{tabular}{|c|c|c|c|c|c|c|}
\hline \multirow{2}{*}{ Características } & \multicolumn{6}{|c|}{ Sistemas de gestión de costes de la calidad en proyectos de construcción } \\
\hline & QPMS & QPTS & QCM & CQCQS & QCPCM & PROMQACS \\
\hline $\begin{array}{c}\text { Modelo genérico } \\
\text { de costes de la } \\
\text { calidad en que } \\
\text { basa }\end{array}$ & Modelo PEF & Modelo PEF & Modelo PEF & Modelo PEF & Modelo PCM & $\begin{array}{c}\text { Modelo de tres } \\
\text { niveles de costes } \\
\text { de fallos } \\
\text { (propuesto) }\end{array}$ \\
\hline $\begin{array}{c}\text { Costes de la } \\
\text { calidad medidos }\end{array}$ & $\begin{array}{c}\text { Prevención, } \\
\text { evaluación y } \\
\text { fallos }\end{array}$ & Fallos & Fallos & Fallos & $\mathrm{COC}$ y $\mathrm{CNOC}$ & Fallos \\
\hline $\begin{array}{c}\text { Alcance de } \\
\text { medición de los } \\
\text { costes de la } \\
\text { calidad }\end{array}$ & $\begin{array}{l}\text { Proyecto } \\
\text { completo }\end{array}$ & $\begin{array}{l}\text { Proyecto } \\
\text { completo }\end{array}$ & $\begin{array}{l}\text { Proyecto } \\
\text { completo }\end{array}$ & $\begin{array}{l}\text { Proyecto } \\
\text { completo }\end{array}$ & $\begin{array}{c}\text { Procesos } \\
\text { seleccionados }\end{array}$ & $\begin{array}{l}\text { Proyecto } \\
\text { completo }\end{array}$ \\
\hline $\begin{array}{l}\text { Tipos de } \\
\text { proyectos en que } \\
\text { fue aplicado }\end{array}$ & $\begin{array}{l}\text { Proyectos } \\
\text { industriales }\end{array}$ & $\begin{array}{l}\text { Proyectos } \\
\text { industriales }\end{array}$ & $\begin{array}{l}\text { Proyectos de } \\
\text { ingeniería civil }\end{array}$ & $\begin{array}{l}\text { No existe } \\
\text { evidencia de } \\
\text { haber sido } \\
\text { probado }\end{array}$ & $\begin{array}{c}\text { Proyectos de } \\
\text { ingeniería civil } \\
\text { y edificación }\end{array}$ & $\begin{array}{l}\text { Proyectos de } \\
\text { ingeniería civil }\end{array}$ \\
\hline $\begin{array}{c}\text { Etapa del } \\
\text { proyecto en la } \\
\text { que se recolectan } \\
\text { los datos }\end{array}$ & $\begin{array}{l}\text { A partir de la } \\
\text { ejecución del } \\
\text { proyecto }\end{array}$ & $\begin{array}{l}\text { A partir de la } \\
\text { ejecución del } \\
\text { proyecto }\end{array}$ & $\begin{array}{l}\text { A partir de la } \\
\text { ejecución del } \\
\text { proyecto }\end{array}$ & $\begin{array}{l}\text { A partir de la } \\
\text { ejecución del } \\
\text { proyecto }\end{array}$ & $\begin{array}{l}\text { A partir de la } \\
\text { ejecución del } \\
\text { proyecto y en } \\
\text { procesos de } \\
\text { gestión }\end{array}$ & $\begin{array}{l}\text { A partir de la } \\
\text { ejecución del } \\
\text { proyecto }\end{array}$ \\
\hline Énfasis & $\begin{array}{l}\text { Reducción de } \\
\text { fallos }\end{array}$ & $\begin{array}{l}\text { Reducción de } \\
\text { fallos }\end{array}$ & $\begin{array}{l}\text { Reducción de } \\
\text { fallos }\end{array}$ & $\begin{array}{l}\text { Reducción de } \\
\text { fallos }\end{array}$ & $\begin{array}{c}\text { Mejora } \\
\text { continua de } \\
\text { procesos }\end{array}$ & $\begin{array}{l}\text { Reducción de } \\
\text { fallos }\end{array}$ \\
\hline
\end{tabular}

la que llamaron: los problemas de ejecución de la planeación y diseño de la gestión de la construcción profesional (Professional Construction Management Planning and Designing Executing Problems) fue creada para analizar las relaciones causales entre estos problemas y su influencia en los objetivos generales de los proyectos.

Por otra parte, Ezeldin y Abu-Ghazala (26) propusieron recientemente un sistema de gestión de la calidad para consultores de diseño, proporcionando otro punto de vista en esta etapa del proyecto. Para esto, plantearon una revisión de las prácticas actuales de diseño de proyectos de edificación residenciales y comerciales. Dicho sistema de gestión de la calidad está basado en elementos de sistemas de gestión de calidad reconocidos en la literatura (TQM, ISO 9000) y en la información proporcionada por expertos en el diseño de proyectos de edificación, de Estados Unidos de América, el Reino Unido, Arabia Saudita, Jordania y Egipto. En relación a estos trabajos recientes $(24,25,26)$ que abordan el diseño en proyectos de construcción, se pueden destacar ciertos puntos que proporcionan un acercamiento a un contexto actual y real de problemas en las prácticas del diseño en proyectos de construcción, tal y como se muestra en la tabla 3. De esta manera, se puede observar que es necesaria una herramienta que permita medir la calidad en los procesos de diseño y, de esta manera, gestionar la mejora continua. Es decir, la aplicación de un modelo de costes de la calidad en el diseño de proyectos de construcción, propor- cionaría una herramienta para la solución de problemas actuales en el diseño de proyectos, tal como se ha demostrado en la presente investigación. La figura 2, nos proporciona un resumen del contexto y de los elementos que actualmente condicionan la aplicación de los sistemas de costes de la calidad en proyectos de construcción, el modelo genérico de costes de la calidad en que se basan dichos sistemas y su aplicación en la fase del proyecto.

\section{CONCLUSIONES}

Se han presentado los sistemas de costes de la calidad desarrollados para proyectos de construcción, sus alcances y limitaciones. Se observó que existe un interés profesional y académico en el desarrollo de modelos de costes de la calidad, pudiéndose encontrar información teórica y práctica. De igual manera, se pueden encontrar datos publicados sobre el empleo de modelos de costes de la calidad, destacando la poca información en relación a la industria de la construcción. La mayoría de las compañías han empleado la tradicional estructura PEF de costes de la calidad. Los sistemas de costes de la calidad desarrollados para proyectos de construcción se aplican a partir de la fase de ejecución del proyecto; el diseño se considera solamente como una causa de fallos. Actualmente, se pueden detectar y generalizar problemas en las prácticas del diseño en proyectos de construcción; destacando la falta de un enfoque sistemático en los procesos del diseño, que permita conducirlos de manera eficiente, sobre todo en la fase del diseño básico; de igual 
Tabla 3

Principales problemas en las prácticas del diseño de proyectos de construcción (basado en Chuang y Tsai, 2005 y Ezeldin y Abu-Ghazala, 2007).

\begin{tabular}{|l|l|}
\hline \multicolumn{1}{|c|}{ Aspecto del diseño } & \multicolumn{1}{|c|}{ Principales problemas que se presentan } \\
\hline Calidad & $\begin{array}{l}\text { No existe un plan definido de aseguramiento de la calidad en } \\
\text { esta etapa. } \\
\text { La calidad en el diseño se relaciona a la cantidad de } \\
\text { información disponible, más no a la eficiencia de la misma; la } \\
\text { calidad no forma parte de un sistema formal definido aplicable } \\
\text { al diseño de cualquier proyecto }\end{array}$ \\
\hline Gestión de recursos & $\begin{array}{l}\text { Una gestión incorrecta de los documentos de diseño. } \\
\text { Una selección incorrecta de los consultores de diseño } \\
\text { Cambios frecuentes durante el diseño. }\end{array}$ \\
\hline Etapas en el diseño & $\begin{array}{l}\text { A pesar de que existe una similitud en las etapas de diseño, no } \\
\text { existe una metodología que permita conducir de manera } \\
\text { sistemática los procesos de diseño del proyecto, sobre todo en } \\
\text { la fase conceptual y preliminar (diseño básico). }\end{array}$ \\
\hline $\begin{array}{l}\text { Solamente para grandes proyectos se aplicaba una revisión } \\
\text { rigurosa. } \\
\text { El control de diseño se lleva a cabo en un porcentaje medio, } \\
\text { mediante listas de comprobación. } \\
\text { Los procesos se controlaban mayormente, mediante registros } \\
\text { hora-hombre y en reuniones. } \\
\text { Las mediciones internas del proceso eran con fines de } \\
\text { certificación mayormente. } \\
\text { Ocasionalmente se aplicaban herramientas o técnicas para } \\
\text { analizar los procesos y medidas de mejora. }\end{array}$ \\
\hline
\end{tabular}

\begin{tabular}{|c|c|c|c|}
\hline $\begin{array}{c}\text { Fase de } \\
\text { aplicación en el } \\
\text { proyecto }\end{array}$ & $\begin{array}{c}\text { Sistemas de gestión de costes de la calidad en } \\
\text { proyectos de construcción }\end{array}$ & Modelo genérico de costes de la calidad \\
\hline \hline Diseño & QC-PCM por Aoieong et al, 2002 & $\begin{array}{c}\text { Modelos de Coste del proceso } \\
\text { (Conformidad + No-conformidad) }\end{array}$ \\
\hline $\begin{array}{c}\text { Ejecución } \\
\text { Entrega y } \\
\text { puesta en } \\
\text { marcha }\end{array}$ & QPMS por Quality Management Task Force, 1987 \\
\hline & QPTS por K. David, 1987 & Modelos de P-E-F \\
(Prevención + Evaluación + Fallos)
\end{tabular}

manera, el seguimiento y control que se hace en esta fase, requiere de mecanismos más definidos. Se considera necesario el desarrollo de herramientas que permita gestionar la calidad en los procesos de diseño de proyectos de construcción, la aplicación de un modelo de costes de la calidad en el diseño de proyectos de construcción sería una alternativa y, de esta manera, se podría gestionar la mejora continua desde las fases iníciales del proyecto. La medición de los costes de la calidad debería ser parte de cualquier programa de gestión de calidad. Con este trabajo se busca mostrar un panorama amplio y actual de la aplicación de los modelos de costes de la calidad y establecer sus limitaciones; así como establecer referencias en el tratamiento de los costes de la calidad en el diseño de proyectos de construcción. La investigación y desarrollo de herramientas y técnicas de gestión de la calidad para la industria de la construcción son necesarias. 


\section{BIBLIOGRAFÍA}

(1) Ahmed, S. M., Aoieong, R. T., Tang, S. L. y Zheng, D. X.M.: "A comparison of quality management systems in the construction industries of Hong Kong and the USA". International Journal of Quality and Reliability Management, Vol. 22, no 2 (2005), pp. 149-161.

(2) Low, S. P. y Yeo, H. K. C.: "A construction quality costs quantifying system for the building industry". International Journal of Quality and Reliability Management, Vol. 15, nº 3 (1998), pp. 329-349.

(3) Gracia V., S. y Dzul L., L.: "Modelo PEF de costes de la calidad como herramienta de gestión en empresas constructoras: una visión actual". Revista Ingeniería de Construcción, Vol. 22, nº 1 (2007), pp. 43-56.

(4) Hwang, G. H. y Aspinwall, E. M.: "Quality cost models and their application: a review". Total Quality Management, Vol. 7, no 3 (1996), pp. 267-281.

(5) Schiffauerova, A. y Thomson, V.: "A review of research on cost of quality models and best practices". International Journal of Quality \& Reliability Management, Vol. 23, nº 6 (2006) pp. 647-669.

(6) Weheba, G. S. y Elshennawy, A. K.: "A revised model for the cost of quality". Journal of Quality and Reliability Management, Vol. 21, $\mathrm{n}^{\circ} 3$ (2004), pp. 291-308.

(7) Climent Serrano, Salvador.: "Los Costes De Calidad Como Estrategia Empresarial: Evidencia Empírica en la Comunidad Valenciana". Tesis Doctoral. España. UV, Facultad de Economía (2003), 523 pp.

(8) Instituto Tecnológico de Galicia.: Proyecto Costes de Calidad en la Construcción (CCCON). (en línea). España, en: http://www.itg.es/proyectos/detalle.php?IdProyecto=28" (Accesado el día 2 de mayo de 2007).

(9) Love, P. E. D. e Irani, Z.: "A project management quality cost information system for the construction industry". Information and Management, Vol. 40 (2003), pp. 649-661.

(10) Aoieong, R. T., Tang, S. L. y Syed, M. A.: "A process approach in measuring quality costs of construction projects: model development". Construction Management and Economics, Vol. 20, $\mathrm{n}^{\circ}$ 2 (2002), pp. 179-192.

(11) Abdul-Rahman, H.: "The cost of non-conformance during a highway project: a case study". Construction Management and Economics, Vol. 13 (1995), pp. 23-32.

(12) CII: "Costs of Quality Deviations in Design and Construction". Quality Management Task Force, Construction Industry Institute, publicación 10 (1) (1989).

(13) Patterson, L., Ledbetter, W.B.: "The Cost of Quality: A Management Tool." Excellence in the Construction Project, American Society of Civil Engineers (1989) pp. 100-105.

(14) Willis, T. H. y Willis, W. D. A quality performance management system for industrial construction engineering projects. International Journal of Quality and Reliability Management, Vol. 13, $n^{\circ} 9$ (1996), pp. 38-48.

(15) Davis, K.: "Measuring Design and Construction Quality Costs". Source Document 30, Construction Industry Institute (1987).

(16) Davis,K., Ledbetter, W.B., Burati, J.L.: "Measuring design and construction quality costs". ASCE Journal of Construction Engineering and Management, Vol. 115 (1989), pp. 389-400.

(17) Abdul-Rahman, H.: "Capturing the cost of quality failures in civil engineering". International Journal of Quality \& Reliability Management, Vol. 10, no 3 (1993), pp. 20-32.

(18) Abdul-Rahman, H.: "Capturing the costs of non-conformance on constructions sities (an application of the quality costs matrix)". International Journal of Quality \& Reliability Management, Vol. 13, $\mathrm{n}^{\circ} 1$ (1996), pp. 48-60.

(19) Hall, M. y Tomkins, C.: "A cost of quality analysis of a building project: towards a complete methodology for design and build". Construction Management and Economics, Vol. 19 (2001) pp. 727-740.

(20) Tang, S. L., Aoieong, R. T.y Ahmed, S. M.: "The use of Process Cost Model (PCM) for measuring quality costs of construction projects: model testing". Construction Management and Economics, Vol. 22, no 3 (2004) pp. 263-275.

(21) Love, P.E.D., y Li, H.: "Quantifying the causes and costs of rework in construction". Construction Management and Economics, Vol. 18 (2000), pp. 479-490

(22) Love, P. E. D. y Sohal, A. S.: "Capturing rework costs in projects". Managerial Auditing Journal, Vol. 8, n 4 (2003), pp. 329-339.

(23) Barber, P., Graves, A., Hall, M., Sheath, D. y Tomkins, C.: "Quality failure costs in civil engineering projects". International Journal of Quality \& Reliability Management, Vol.17 (2000), pp. 479-492.

(24) Chuang, C.-C. y Tsai, C.-C.: "A fuzzy neural approach for diagnosing PCM executing problem". Proceedings of the 2005 Systems and Information Engineering Design Symposium (2005), pp.171-176.

(25) Wang, Ching-Hwang; Tsai, Chia-Chang y Cheng, Yi-Yen.: "Knowledge-based diagnosis model for PCM executing problems in public construction". Construction Management and Economics, Vol. $25, n^{\circ} 2$ (2007), pp. 129-142.

(26) Ezeldin, A. S. y Abu-Ghazala, H.: "Quality Management System for Design Consultants: Development and Application on Projects in the Middle East". Journal of management in engineering, Vol. 23, $\mathrm{n}^{\circ}$ (2) (2007), pp. 75-87. 